\title{
Lack of cross-desensitization between leptin and prolactin signaling pathways despite the induction of suppressor of cytokine signaling 3 and PTP-1B
}

\author{
A F Roy, Y Benomar, V Bailleux, C M Vacher, A Aubourg, A Gertler ${ }^{1}$, J Djiane and M Taouis \\ Neuroendocrinologie Moléculaire de la Prise Alimentaire, UMR 1197 INRA/Université Paris XI, IBAIC Bât. 447, Orsay 91405, France \\ ${ }^{1}$ Faculty of Agricultural, Food and Environmental Quality Sciences, The Institute of Biochemistry, Food Science and Nutrition, The Hebrew University of \\ Jerusalem, PO Box 12, Rehovot 76100, Israel \\ (Correspondence should be addressed to M Taouis; Email: mohammed.taouis@u-psud.fr)
}

\begin{abstract}
Hyperprolactinemia and hyperleptinemia occur during gestation and lactation with marked hyperphagia associated with leptin resistance. Prolactin (PRL) induces the expression of orexigenic neuropeptide $\mathrm{Y}$ (NPY) through the activation of JAK-2/STAT-3 signaling pathway in hypothalamic paraventricular nucleus (PVN) leading to hyperphagia. PRL may also act through the inhibition of anorexigenic effect of leptin via induction of suppressor of cytokine signaling 3 (SOCS-3). This paper aimed to co-localize PRL (PRL-R) and leptin (ObRb) receptors in the hypothalamus of female rats and investigate the possible cross-desensitization between PRL-R and ObRb. We showed that: 1) PRL-R and ObRb are expressed in the PVN and co-localized in the same neurons; 2) in lactating females leptin failed to activate JAK-2/STAT-3 signaling pathway; 3 ) in Chinese Hamster Ovary (CHO) stably co-expressing PRL-R and $\mathrm{ObRb}$, overexposure to PRL did not affect leptin signaling
\end{abstract}

but totally abolished PRL-dependent STAT-5 phosphorylation. The overexposure to leptin produces similar results with strong alteration of leptin-dependent STAT-3 phosphorylation, whereas PRL-dependent STAT-5 was not affected; and 4) $\mathrm{CHO}-\mathrm{ObRb} / \mathrm{PRL}-\mathrm{R}$ cells overexposure to leptin or PRL induces the expression of negative regulators SOCS-3 and PTP1B. Thus, we conclude that these negative regulators affect specifically the inducer signaling pathway; for instance, SOCS-3 induced by PRL will affect PRL-R signaling but not ObRb signaling and vice versa. Finally, the lack of cross-desensitization between PRL-R and ObRb suggests that hyperphagia observed during gestation and lactation may be attributed to a direct effect of PRL on NPY expression, and is most likely exacerbated by the physiological leptin resistance state.

Journal of Endocrinology (2007) 195, 341-350

\section{Introduction}

During pregnancy and lactation, increased appetite and food intake anticipates the changes in metabolic demand of these physiological conditions (Rosso 1987, Grattan et al. 2001). Maternal homeostatic regulators are set at new levels to allow the mother to cope with metabolic physiological demand of pregnancy and lactation. This adaptive state is driven by hormonal changes that take place in the brain of the mother. Prolactin (PRL) and leptin are among the hormones that show significant plasma level changes during these periods. During pregnancy and lactation, other hormones may also contribute to hyperphagia. The lack of central oxytocin action may partly contribute to maternal hyperphagia (Douglas et al. 2007). Progesterone also stimulates appetite and food intake in the female in the presence of elevated leptin (Rosso 1987, Grueso et al. 2001).

Leptin, the $16 \mathrm{kDa}$ peptide hormone product of the ob gene, is mainly produced by adipose tissue and acts at the hypothalamus to decrease food intake. Leptin also plays key roles in reproduction such as puberty onset and ovarian, testicular, and uterine functions (Chehab et al. 1996, Hoggard et al. 1997, Zamorano et al. 1997, Kitawaki et al. 2000). During pregnancy, plasma leptin levels at mid-gestation and so on are high when compared with non-pregnant females (Kawai et al. 1997, Tomimatsu et al. 1997, Lewandowski et al. 1999) associated with increased or unchanged food intake, suggesting a leptin resistance state (Shirley 1984, Rosso 1987). Moreover, recent studies show that ObRb mRNA levels in the ventromedial hypothalamic nucleus (VMH) are significantly reduced during pregnancy, and that levels of leptininduced STAT-3 phosphorylation were specifically suppressed in the $\mathrm{VMH}$ and arcuate nucleus (ARC) of pregnant rats compared with non-pregnant rats (Grattan et al. 2007). The source of hyperleptinemia during pregnancy originates in humans from the placenta and adipose tissue (Masuzaki et al. 1997), and in rodents from the adipose tissue (Kawai et al. 1997, Tomimatsu et al. 1997). During lactation, plasma leptin levels return to the levels observed in non-pregnant rat females (Seeber et al. 2002) or reduce by about 50\% compared with non-lactating animals (Brogan et al. 1999, 
Denis et al. 2003). In pregnant women, plasma leptin levels are maintained high (Mukherjea et al. 1999).

PRL plays a crucial role during pregnancy and lactation by exerting numerous effects on the central nervous system such as induction of maternal behavior, stimulation of oxytocin neurons, increasing appetite and food intake, suppression of adrenocorticotrophin secretion in response to stress, and suppression of fertility. In addition, PRL is involved in lobuloalveolar development of the mammary gland and differentiation of mammary cells (Bole-Feysot et al. 1998). PRL plasma levels are maintained high during late pregnancy by reduced tonic inhibitory influence of tuberoinfundibular dopamine neurons, and during lactation where suckling exerts a strong stimulatory effect on PRL release (Freemark 2001, Grattan 2002).

During the late pregnancy period, females show increased leptin and PRL plasma levels along with increased food intake suggesting that leptin fails to exert its anorexic action leading to leptin resistance. Furthermore, in virgin female rats, PRL significantly increases food intake when injected into the paraventricular nucleus (PVN; Sauvé \& Woodside 2000, Woodside 2007). Thus, PRL may have a direct effect on food intake through NPY expression in hypothalamic dorsomedian nucleus, where NPY-positive neurons express PRL-R mRNA (Chen \& Smith 2004). It has also been shown that during lactation the expression of NPY raised in various locations of the hypothalamus such as arcuate and dorsomedial nuclei (Chen \& Smith 2004).

Taken together, these data suggest that the orexigenic effect of PRL is predominant and attenuates the anorexic effect of leptin in the hypothalamus during late pregnancy. The effect of PRL may be attributed to a direct effect on the expression of NPY or through the inhibition of leptin action.

In our study, we focused mainly on PRL and leptin. These cytokines share a number of signaling pathways acting through cytokine class I receptors. Long isoforms of PRL and leptin receptors $(\mathrm{ObRb})$ act respectively through JAK-2/STAT-5 and JAK-2/STAT-3 signaling pathways (Bjorbaek et al. 1997, Bole-Feysot et al. 1998). Both pathways induce the expression of suppressor of cytokine signaling 3 (SOCS-3), which is a negative regulator of both hormones (Ihle 1995). We hypothesize that during pregnancy, due to high PRL plasma levels, PRL activates the PRL-R/JAK-2/ STAT-5 pathway and induces SOCS-3 resulting in inhibiting leptin action and subsequent increase in food intake. In the present study, we first showed that both PRL-R and ObRb receptors are co-expressed in the same neurons in hypothalamic areas involved in the control of food intake such as the PVN. Secondly, to investigate a possible cross-talk between PRL-R and ObRb, Chinese Hamster Ovary (CHO) cells were stably co-transfected with cDNAs encoding for both receptors. In co-transfected cells, PRL pre-treatment did not affect ObRb signaling despite SOCS-3 induction and vice versa. Thus, PRL and leptin downregulate their own signaling with no cross-desensitization.

\section{Materials and Methods}

\section{Animals}

Lactating and virgin female rats were housed under a $12 \mathrm{~h}$ light:12 $\mathrm{h}$ darkness cycle with access to food and water ad libitum and used in accordance with French and European ethics authorities and local regulations.

\section{Tissues preparation for brain slice experiments}

Four virgin and lactating female rats were rapidly decapitated without anesthesia, and their brains removed immediately and transferred into ice-cold oxygenated Krebs-Henseleit buffer (Sigma) added to $25 \mathrm{mM} \mathrm{NaHCO}, 2 \mathrm{mM} \mathrm{CaCl}, 11 \mathrm{mM}$ glucose, $60 \mathrm{mM}$ ascorbic acid, and $31.5 \mathrm{mM}$ thiourea. Three hundred micrometers thick hypothalamus slices were prepared and warmed to $35.5{ }^{\circ} \mathrm{C}$ in Krebs bicarbonate buffer. After 30-min incubation, $1 \mu \mathrm{g} / \mathrm{ml}$ leptin or PRL were added in the incubation medium for $10 \mathrm{~min}$. By micropunch, the arcuate nucleus were recuperated and incubated in an ice-cold lysis buffer $(20 \mathrm{mM}$ Tris- $\mathrm{HCl}$ (pH 7-5), 1\% Nonidet P-40, 1\% Triton X-100, 10\% glycerol, $150 \mathrm{mM} \mathrm{NaCl}, 1 \mathrm{mM}$ EGTA, $1 \mathrm{mM}$ EDTA, protease inhibitors $(0.35 \mathrm{mg} / \mathrm{ml}$ phenylmethylsulfonyl fluoride (PMSF), $2 \mu \mathrm{g} / \mathrm{ml}$ leupeptin, $2 \mu \mathrm{g} / \mathrm{ml}$ aprotinin), and phosphatase inhibitors $\left(1 \mathrm{mM} \mathrm{Na} \mathrm{VO}_{4}, 10 \mathrm{mM}\right.$ sodium fluoride, $20 \mathrm{mM}$ sodium $\beta$-glycerophosphate, $10 \mathrm{mM}$ benzamidine)), and then sonicated (Elmasonic, Fisher Bioblock Scientific, Illkirch, France) for $10 \mathrm{~min}$. Lysates were clarified by centrifugation at $15300 \boldsymbol{g}$ for $40 \mathrm{~min}$ and supernatants kept for western blot analyses. Protein concentrations of supernatants were determined using a protein assay kit (Pierce, Perbio Science, France).

\section{Cell culture: stable and transient transfections}

CHO cells were grown in HAM-F12 supplemented with 10\% fetal calf serum (FCS), $100 \mathrm{U} / \mathrm{ml}$ penicillin/streptomycin, and $100 \mathrm{U} / \mathrm{ml}$ glutamine at $37^{\circ} \mathrm{C}$ in $5 \% \mathrm{CO}_{2}$. The cDNA encoding mouse $\mathrm{ObRb}$ and PRL-R were produced as described previously (Edery et al. 1989, Bjorbaek et al. 1997). Cells were double transfected with ObRb and PRL-R expression vectors carrying geneticin and hygromycin resistance respectively. Selected double-transfected cells (CHO-ObRb/PRL-R) were cultured in a medium containing both antibiotics.

$\mathrm{CHO}$ cells stably expressing ObRb were transiently transfected with various concentrations of PRL-R expression vector DNA $(1-5 \mu \mathrm{g})$.

\section{Cell stimulation and western blot analysis}

Cells were grown in $10 \mathrm{~cm}$ dishes and serum deprived for $16 \mathrm{~h}$ before stimulation with hormones. Acute and chronic PRL and leptin treatments were performed on CHO-ObRb/PRL-R. For acute stimulations, cells were incubated in serum-free medium in the presence or absence of various concentrations (as indicated in the figures) of leptin or PRL for $10 \mathrm{~min}$. In the 
kinetic studies, cells were incubated in the absence or presence of $500 \mathrm{ng} / \mathrm{ml}$ leptin or PRL for various time periods. For chronic treatment, cells incubated in serum-free medium in the absence or presence of $500 \mathrm{ng} / \mathrm{ml}$ leptin or PRL for $16 \mathrm{~h}$, then washed, and acutely stimulated for $10 \mathrm{~min}$ by leptin or PRL. Following these treatments, cells were harvested by rinsing in ice-cold PBS and scraping into $500 \mu \mathrm{l}$ ice-cold lysis buffer $(20 \mathrm{mM}$ Tris-HCl (pH 7·5), 1\% Nonidet P-40, 1\% Triton X-100, 10\% glycerol, $150 \mathrm{mM} \mathrm{NaCl}, 1 \mathrm{mM} \mathrm{MgCl}_{2}, 1 \mathrm{mM} \mathrm{CaCl}_{2}$, protease inhibitors (0.35 mg/ml PMSF, $2 \mu \mathrm{g} / \mathrm{ml}$ leupeptin, $2 \mu \mathrm{g} / \mathrm{ml}$ aprotinin), and phosphatase inhibitors $\left(1 \mathrm{mM} \mathrm{Na} \mathrm{VO}_{4}, 10 \mathrm{mM}\right.$ sodium fluoride, $20 \mathrm{mM}$ sodium $\beta$-glycerophosphate, $10 \mathrm{mM}$ benzamidine)). Lysates were clarified by centrifugation at $15300 \mathrm{~g}$ for $40 \mathrm{~min}$ and supernatants kept for western blot analyses. Protein concentrations of supernatants were determined using a protein assay kit (Pierce). Cell proteins $(30 \mu \mathrm{g})$ were resolved in SDS-PAGE followed by western blot using adequate antibodies (as indicated in the Results section). Then, western blot bands were revealed by enhanced chemiluminescence (ECL).

\section{Immunohistochemistry}

Three female rats were anesthetized with sodium pentobarbital $(25 \mathrm{mg} / \mathrm{kg})$. Brain fixation and sectioning were performed as described previously (Vacher et al. 2002). Coronal brain sections $(50 \mu \mathrm{m})$ were cut on a vibroslicer (1000Plus, Harvard Apparatus, Holliston, MA, USA) and blocked for endogenous peroxidase in $1 \% \mathrm{H}_{2} \mathrm{O}_{2}$. Sections were incubated for $24 \mathrm{~h}$ at $4{ }^{\circ} \mathrm{C}$ with mouse anti-PRL-R (1:200; Affinity BioReagents, Golden, CO, USA) or rabbit anti-ObRb prepared in our laboratory, and revelations performed as described previously (Vacher et al. 2002). Control experiments were performed by omitting the primary antibodies. For the double immunofluorescence procedure, coronal $50 \mu \mathrm{m}$ thick sections were incubated simultaneously with mouse anti-PRL-R and rabbit antiObRb antibodies for $24 \mathrm{~h}$ at $4{ }^{\circ} \mathrm{C}$. The simultaneous visualization of the two primary antisera was performed with Fluor Probes 488 conjugated donkey anti-rabbit and tetra methylrhodamine isothiocyanate (TRITC)-conjugated donkey anti-mouse immunoglobulins (1:400). After washing, brain sections were mounted with Vectashield. Double immunofluorescence was investigated using an ArgonKrypton laser (Bio-Rad MRC 1024 ES laser, Marnes la Cognette, France) scanning confocal microscope coupled to a Nikon Diaphot 300.

Cross-over fluorescence can be ruled out, as spectra of both fluorochromes do not overlap. Each optical section $(1 \mu \mathrm{m})$ was averaged thrice. The free software ImageJ (http://rsb.info.nih. gov/ij) was used to work captured pictures. In the case of double immunohistochemistry, figures resulted due to the projection of 13 successive optical sections, and in the case of zoom optical, projection of 10 sections. Co-localization pictures were the combination of each corresponding fluorescent signal.

\section{Chemicals}

The ObRb expression vector was a generous gift from $\mathrm{Dr}$ Christian Bjorbaeck. Ovine leptin was produced as described previously (Gertler et al. 1998). Antibodies directed toward total STAT-3 and 5, phospho-STAT-3 and 5 were obtained from Cell Signaling (Ozyme, St Quentin en Yvelines, France). The ECL detection system was from Amersham. Fluor Probes 488 conjugated anti-rabbit was from Interchim (Montluçon, France). TRITC-conjugated anti-mouse and anti-rabbit $\operatorname{IgG}$ conjugated to horseradish peroxidase were from Sigma. Vectashield was from Vector Laboratories (Burlingame, CA, USA).

\section{Results}

Leptin resistance in lactating female rats

To determine leptin sensitivity of hypothalamic arcuate nucleus, hypothalamic slices of virgin and lactating female rats were incubated in the presence or absence of leptin $(1 \mu \mathrm{g} / \mathrm{ml})$ for $10 \mathrm{~min}$. Following the western blot analysis, leptin significantly induced STAT-3 phosphorylation in virgin females, whereas in lactating females, this was completely abolished (Fig. 1).

A

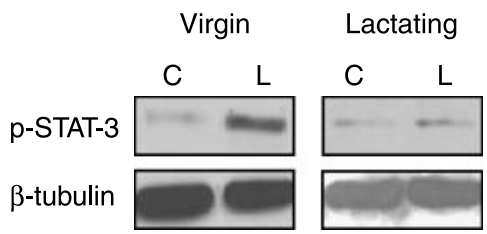

B

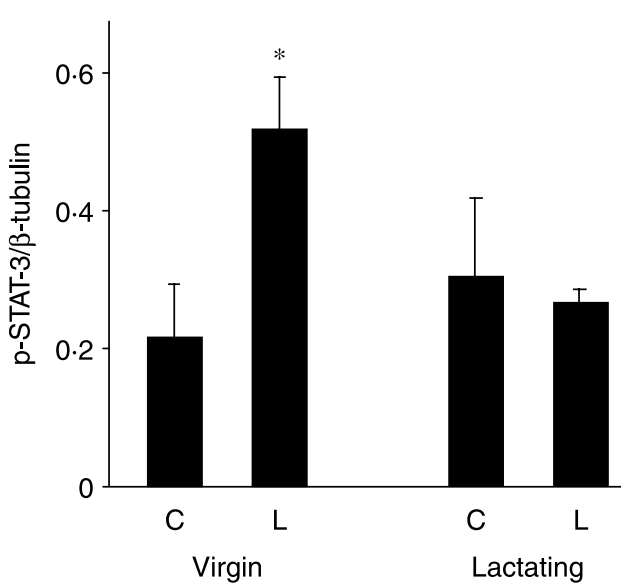

Figure 1 Hypothalamic leptin-dependent STAT-3 phosphorylation in virgin and lactating female rats. In A, arcuate nuclei of virgin and lactating females are incubated for $10 \mathrm{~min}$ with $1 \mu \mathrm{g} / \mathrm{ml}$ leptin. Mean results of four independent experiments, expressed as mean \pm S.E.M. (B), are represented; $* P<0 \cdot 05$.

Journal of Endocrinology (2007) 195, 341-350 

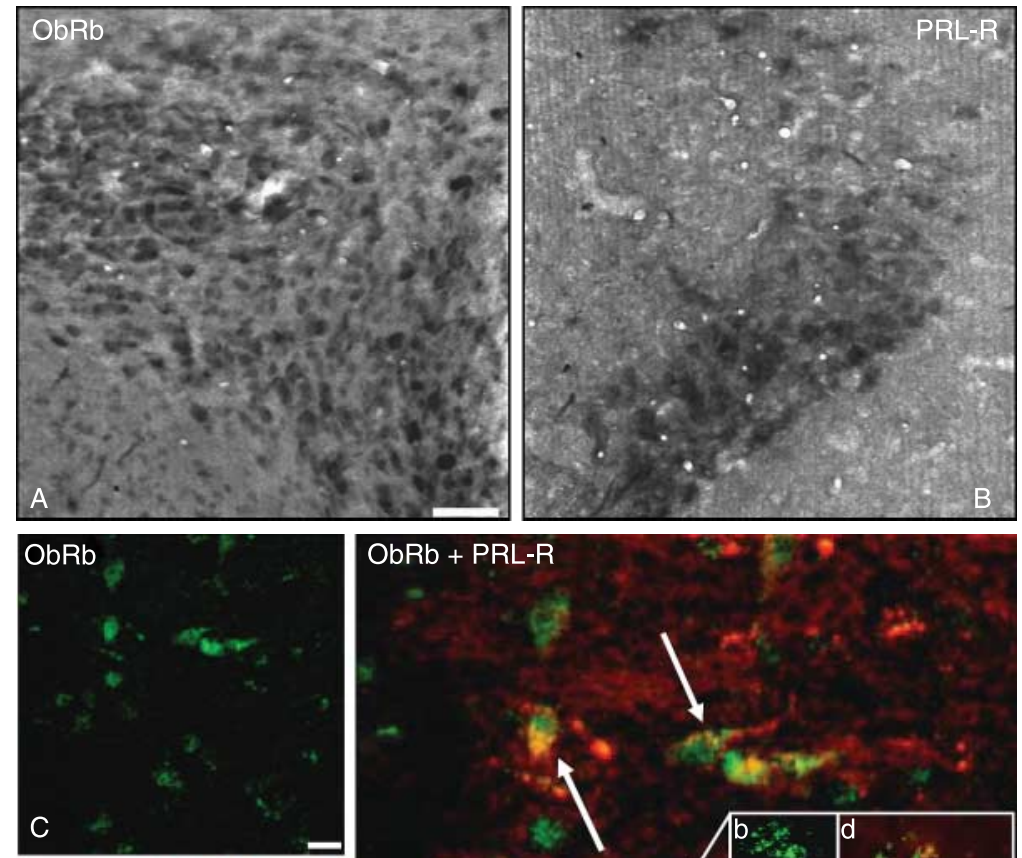

\section{$-\mathrm{R}$}
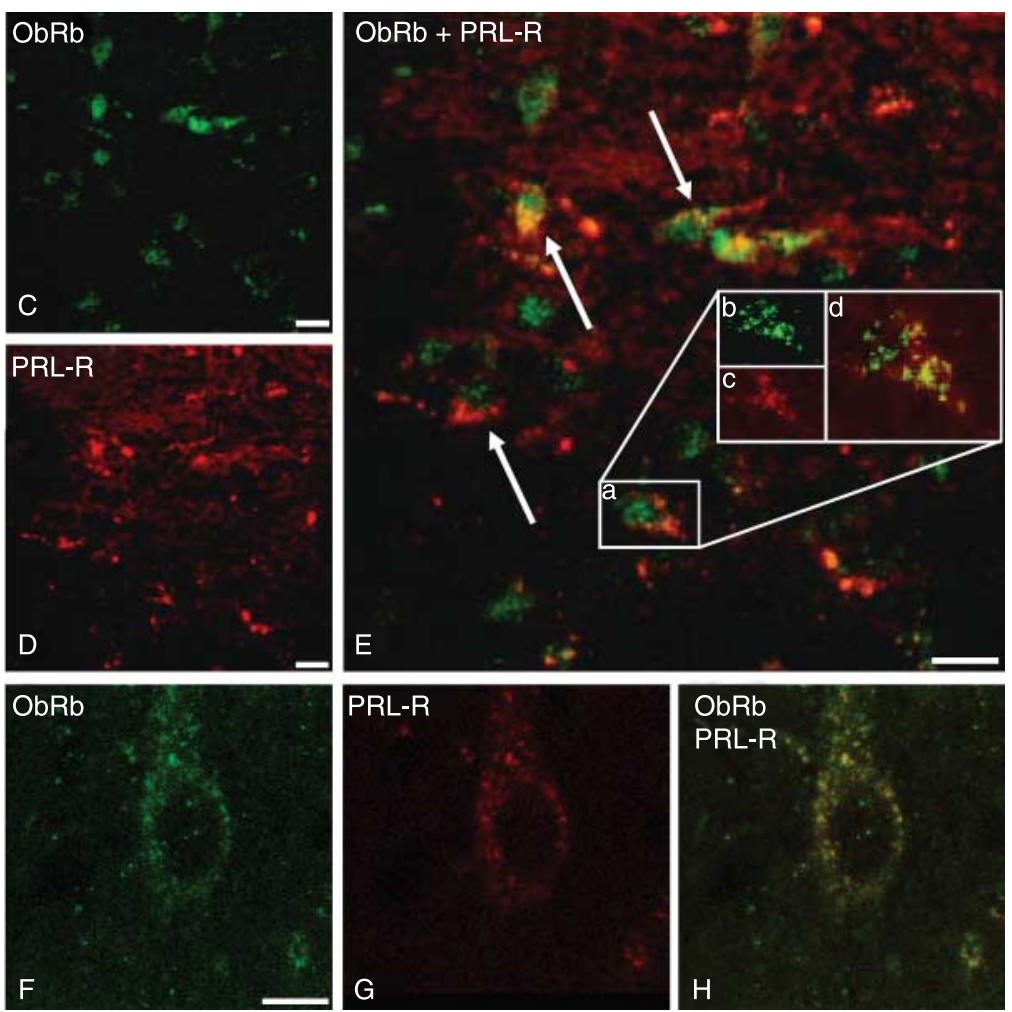

Figure 2 Leptin $(\mathrm{ObRb})$ and prolactin (PRL-R) receptor localization in female rat paraventricular nucleus. Immunohistochemical analysis shows $\mathrm{ObRb}(\mathrm{A})$ and PRL-R (B) immunoreactivities in the magnocellular portion of the paraventricular nucleus of the female rat. An example of double immunofluorescent staining for ObRb and PRL-R is shown (E and $H)$. The images using the confocal microscopy are displayed with the $z$-axis (z-stacked images) where arrows indicate cells co-expressing both ObRb and PRL-R (E). Green fluorescence reveals ObRb immunoreactivity ( $\mathrm{C}$ and F), while red fluorescence represents the presence of PRL-R (D and G). $F$ to $H$ represent an optical zoom of one neuron. The insert a (z-stacked image) in $E$ represents one neuron with co-localization, the inserts $b$ $(\mathrm{ObRb}), \mathrm{c}(\mathrm{PRL}-\mathrm{R})$, and $d(\mathrm{ObRb}$ and PRL-R) illustrate a focal plan (in insert $\mathrm{a}, \mathrm{x}$ and $\mathrm{y}$ field) of this neuron. Scale bars: $60 \mu \mathrm{m}(\mathrm{A}$ and $\mathrm{B})$ and $10 \mu \mathrm{m}(\mathrm{C}-\mathrm{H})$.

\section{Co-localization of $\mathrm{ObR} b$ and $P R L-R$ in the hypothalamic $P V N$}

In the PVN, ObRb-immunoreactive cells occupied the major part of the nucleus (Fig. 2A), both in parvocellular and magnocellular neurons. This staining was primarily observed in cell bodies. Confocal microscopy scanning at higher magnification revealed a punctiform staining throughout the cytoplasm of neurons (Fig. 2C and F). However, PRL-Rimmunopositive cells were mostly observed in the peripheral part of the PVN (Fig. 2B), namely in magnocellular neurons. PRL-R immunoreactivity exhibited a somatic punctiform staining similar to that of ObRb (Fig. 2D 
and $G$ ). The double immunostaining experiments revealed a co-localization of $\mathrm{ObRb}$ and PRL-R in the magnocellular neurons (Fig. 2E and $\mathrm{H}$ ). Arrows in Fig. 2E indicate cells co-expressing both ObRb and PRL-R. The insert $d$ in panel $\mathrm{E}$ represents a focal plan (in insert $\mathrm{a}, \mathrm{x}$ and $\mathrm{y}$ field) of one neuron (insert a) and illustrates co-localization of ObRb (insert b) and PRL-R (insert c).

\section{Stable transfection of $O b R b$ and PRL-R in CHO cells (CHO-ObRb/PRL-R)}

To determine the possible cross-regulation between leptin and PRL signaling pathways, CHO cells were stably transfected with ObRb and PRL-R cDNAs. The functionality of both receptors was evaluated by the measurement of STAT- 3 and 5 phosphorylation in response to leptin and PRL stimulations respectively. Leptin induced the phosphorylation of STAT-3 in a dose-dependent manner and the phosphorylation was detected already with $5 \mathrm{ng}$ leptin/ml (Fig. 3A). The dosedependent effect was assessed in three separate experiments and the mean results are presented in Fig. 3B. In addition, when $500 \mathrm{ng}$ leptin/ml were used, the kinetics of STAT-3 phosphorylation revealed a peak at $10 \mathrm{~min}$ after incubation and then gradually decreased (Fig. 3C). The mean results indicate that leptin significantly induced STAT-3 phosphorylation levels from 5 to $30 \mathrm{~min}$, then decreased, and returned to the basal level (Fig. 3D). Similar results were obtained for STAT-5 phosphorylation in response to PRL. PRL induced a dose-dependent effect with a significant increase in STAT-5 phosphorylation with 250 and $500 \mathrm{ng}$ PRL/ml (Fig. 4A and
B). The kinetics of STAT- 5 phosphorylation in response to PRL reached its maximum after 5-10 min of incubation and then declined to reach basal values (Fig. 4C and D).

\section{Impact of chronic PRL or leptin treatment on CHO-ObRb/PRL-R cells sensitivity to both hormones}

To investigate the possible cross-regulation between $\mathrm{ObRb} /$ JAK-2/STAT-3 and PRL-R/JAK-2/STAT-5 signaling pathways, $\mathrm{CHO}-\mathrm{ObRb} / \mathrm{PRL}-\mathrm{R}$ cells were pre-treated with or without leptin $(500 \mathrm{ng} / \mathrm{ml})$ or PRL $(500 \mathrm{ng} / \mathrm{ml})$ for various time periods ( $0 \mathrm{~h}, 1 \mathrm{~h}, 2 \mathrm{~h}, 4 \mathrm{~h}$, and $16 \mathrm{~h})$ and then stimulated for $10 \mathrm{~min}$ with either PRL or leptin. The western blot analyses of cell lysates were performed by anti-phospho-STAT-3 or 5 antibodies. Results were normalized to either total STAT-3 or 5 .

In leptin pre-treated cells, leptin-dependent phosphorylation of STAT-3 was completely abolished from $1 \mathrm{~h}$ of pretreatment (Fig. 5A and B). In contrast, PRL-dependent phosphorylation of STAT-5 was not affected and remained significantly high independent of leptin pre-treatment duration (Fig. 5C and D).

In PRL pre-treated cells, PRL-dependent phosphorylation of STAT-5 was completely abolished from $1 \mathrm{~h}$ of pretreatment (Fig. 6A and B), whereas leptin-dependent STAT-3 phosphorylation was not affected at all by PRL pre-treatment (Fig. 6C and D).

To determine whether the absence of cross-regulation between PRL-R and ObRb was attributed to an inadequate $\mathrm{ObRb} / \mathrm{PRL}-\mathrm{R}$ ratio expressed on $\mathrm{CHO}$ cells,
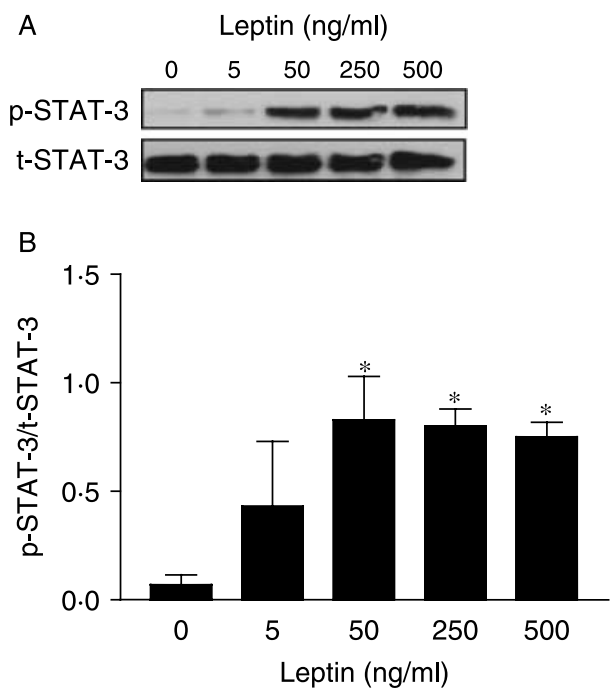

C

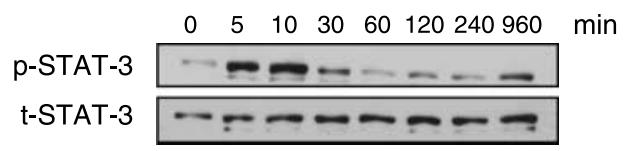

D

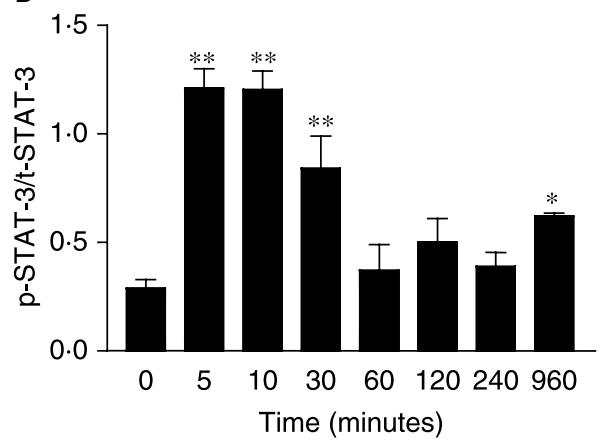

Figure 3 Stably transfected $\mathrm{CHO}$ cells express functional ObRb and PRL-R. CHO cells stably co-expressing $\mathrm{ObRb}$ and PRL-R were serum starved and subjected to either a dose- or time-dependent effect of leptin. In A, cells were subjected to increased concentrations of leptin for $10 \mathrm{~min}$ and STAT-3 phosphorylation was determined by western blot. Mean results of three independent experiments, expressed as mean \pm s.E.M. (B), are presented; ${ }^{*} P<0 \cdot 05$. In C, cells were subjected to single concentration $(500 \mathrm{ng} / \mathrm{ml})$ of leptin and STAT-3 phosphorylation was determined by western blot at various incubation time periods. Mean results of three independent experiments are expressed as mean \pm S.E.M. (D); ${ }^{* *} P<0 \cdot 005$. 


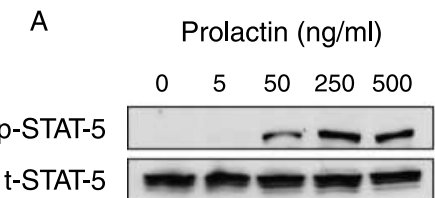

B

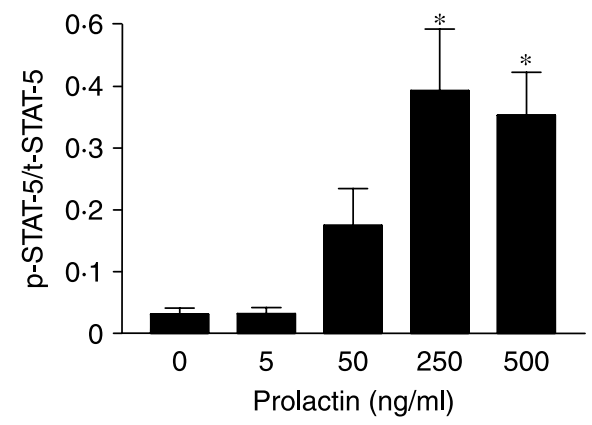

C Prolactin $(500 \mathrm{ng} / \mathrm{ml})$

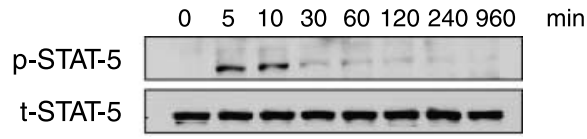

D

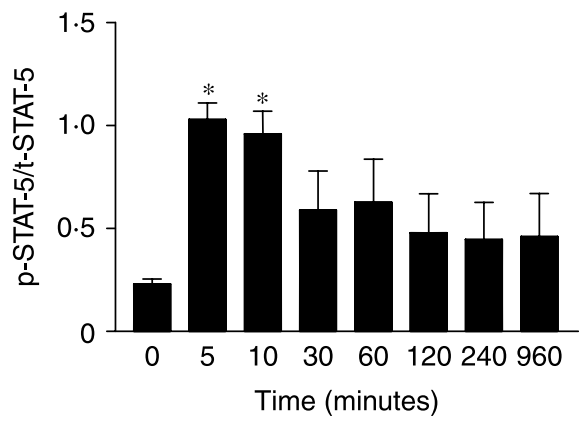

Figure 4 Stably transfected $\mathrm{CHO}$ cells express functional ObRb and PRL-R. CHO cells stably co-expressing $\mathrm{ObRb}$ and PRL-R were serum starved and subjected to either a dose-dependent or time-dependent effect of leptin. In A, cells were subjected to increased concentrations of PRL, and STAT-5 phosphorylation was determined by western blot; this was performed in three independent experiments and mean results are expressed as mean \pm s.E.M. (B); ${ }^{*} P<0 \cdot 05$. In C, cells were subjected to single concentration $(500 \mathrm{ng} / \mathrm{ml})$ of PRL, and STAT-5 phosphorylation was determined by western blot at various incubation time periods. This experiment was performed in triplicate, and mean results are expressed as mean \pm S.E.M. (D); ${ }^{*} P<0 \cdot 05$.

$\mathrm{CHO}-\mathrm{ObRb}$ cells stably expressing $\mathrm{ObRb}$ were transiently transfected with increasing amount of PRL-R cDNA $(1-5 \mu \mathrm{g} / \mathrm{dish})$. In these cells, STAT-5 phosphorylation was estimated after PRL stimulation. PRL increased STAT-5 phosphorylation in a PRL-R cDNA dose-dependent

A

Leptin pre-treatment

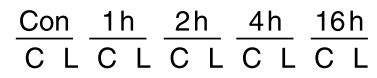

p-STAT-3

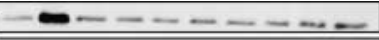

t-STAT-3 manner (Fig. 7A). STAT-3 phosphorylation was estimated in response to leptin following PRL pre-treatment. Despite PRL pre-treatment, leptin clearly increased STAT-3 phosphorylation independent of transfected amount of PRL-R cDNA (Fig. 7B).

C

Leptin pre-treatment

Con $1 \mathrm{~h}$ 2h $4 \mathrm{~h} 16 \mathrm{~h}$

C P C P C P C P C P

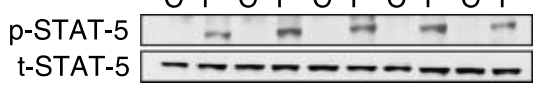

B

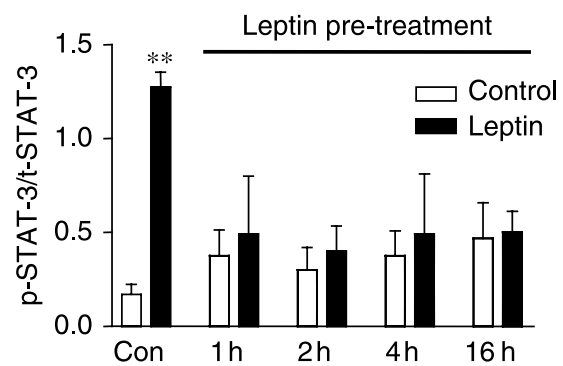

D

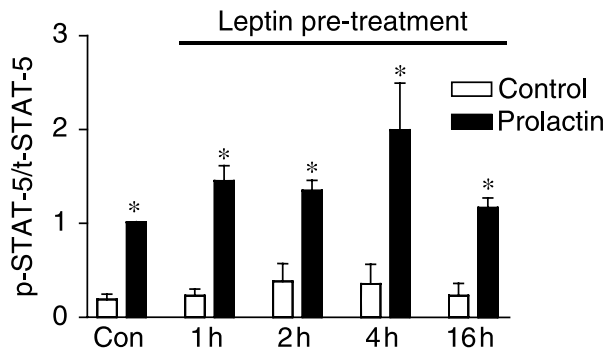

Figure 5 Impact of chronic leptin treatment on ObRb and PRL-R signaling pathways. CHO cells stably co-expressing ObRb and PRL-R were starved and pre-treated with leptin $(500 \mathrm{ng} / \mathrm{ml})$ for various time periods and then acutely stimulated (10 min) with leptin (A and B) or PRL (C and D). STAT-3 and 5 phosphorylation were determined by western blot and normalized to total STAT-3 or 5. B and D present the mean results of three independent experiments and expressed as mean \pm S.E.M.; $* P<0 \cdot 05$ and ${ }^{* *} P<0 \cdot 005$. 
A
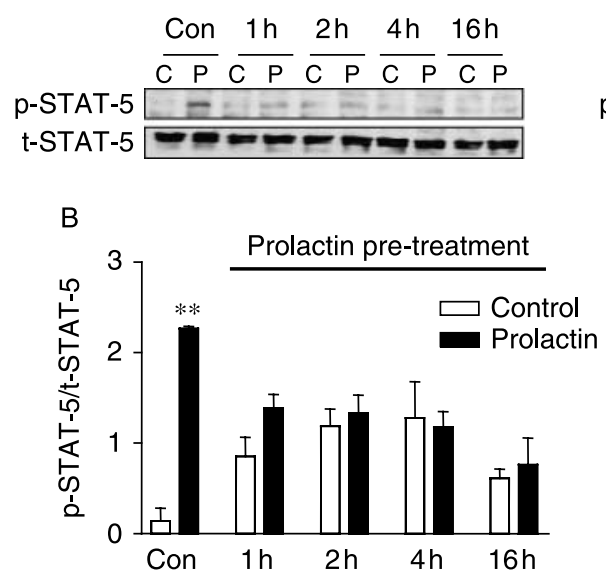

C Prolactin pre-treatment

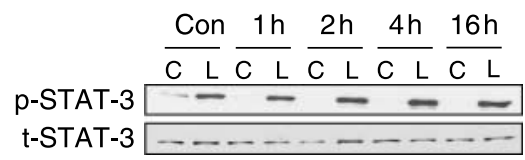

D

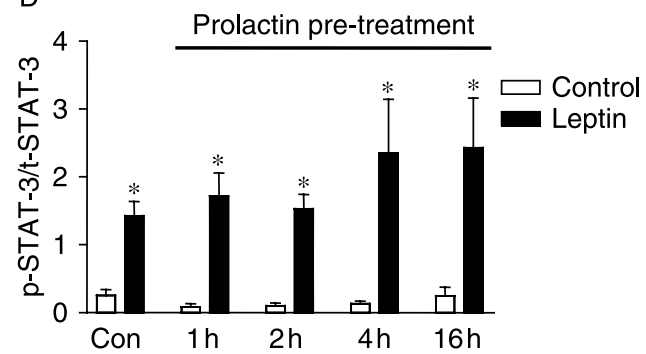

Figure 6 Impact of chronic prolactin treatment on ObRb and PRL-R signaling pathways. CHO cells stably co-expressing ObRb and PRL-R were starved and pre-treated with PRL $(500 \mathrm{ng} / \mathrm{ml})$ for various time periods and then acutely stimulated $(10 \mathrm{~min})$ with PRL (A and B) or leptin (C and D). STAT-5 and 3 phosphorylation were determined by western blot and normalized to total STAT- 5 or 3 . B and D present the mean results of three independent experiments and are expressed as mean \pm S.E.M.;

$* P<0 \cdot 05$ and ${ }^{* *} P<0 \cdot 005$.

The lack of cross-regulation between $O b R b$ and $P R L-R$ does not involve SOCS-3 nor PTP-1B

The absence of cross-regulation between leptin and PRL signaling pathways in $\mathrm{CHO}-\mathrm{ObRb} / \mathrm{PRL}-\mathrm{R}$ cells has raised a question concerning the induction of the common negative regulators SOCS-3 and PTP-1B. To evaluate these inductions, cells were incubated for various time periods with leptin and/or PRL (1-16 h), and then SOCS-3 and PTP-1B were estimated by western blot using specific antibodies. Treatment with leptin or PRL induced SOCS-3 expression, and this expression remained significantly high over $16 \mathrm{~h}$ of incubation (Fig. 8A and B). PTP-1B was also significantly induced by both leptin and PRL from $1 \mathrm{~h}$ of treatment (Fig. 8C and D).

\section{Discussion}

Significant changes occur in hormonal status and energy intake in mammalian females during gestation and lactation, as are mirrored by hyperprolactinemia, hyperleptinemia, and hyperphagia (Garcia et al. 2000, Grattan 2002). It has been suggested that hyperprolactinemia and hyperphagia are most likely linked through the activation of the orexigenic neuropeptide Y (NPY) expression by PRL in several hypothalamic nuclei involved in the control of food intake, and specifically in the PVN (Chen \& Smith 2004). Several reports have also demonstrated that leptin levels are high in serum during human and rodent gestation (Chien et al. 1997, Lage et al. 1999). During lactation, leptinemia is maintained at the levels observed in non-pregnant females (Kawai et al. 1997). Thus, the resulting hyperphagia observed during gestation and lactation indicates a physiological state of leptin resistance in the hypothalamus. In this hormonal environment, the hyperphagic behavior could also be attributed to hyperprolactinemia through the induction of NPY expression. In the present paper, we attempted to understand the possible cross-talk between PRL-R and ObRb signaling pathways to establish whether the leptin resistance observed during gestation and lactation results from the inhibition of ObRb signaling pathways by PRL.
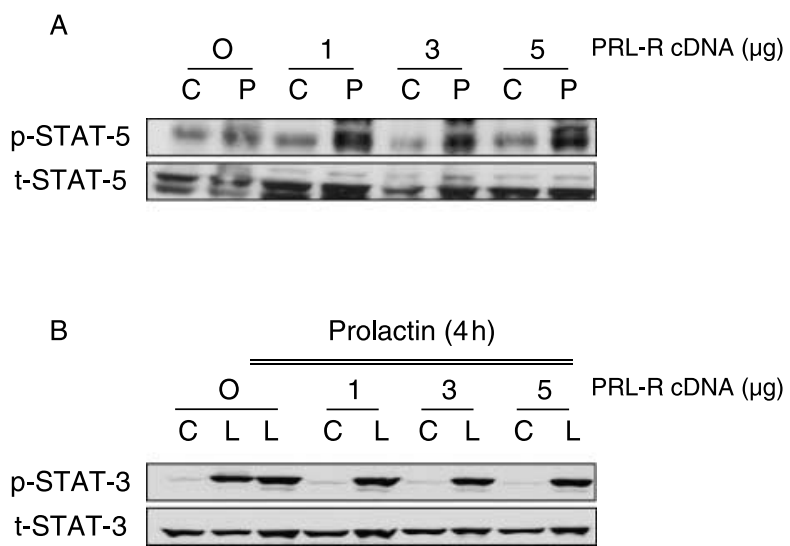

Figure 7 Leptin-dependent STAT-3 phosphorylation in $\mathrm{CHO}-\mathrm{ObRb}$ cells transiently expressing various amounts of PRL-R. CHO cells stably transfected with cDNA encoding ObRb were transiently transfected with increased amounts of PRL-R cDNA $(0-5 \mu \mathrm{g})$. STAT- 5 and 3 phosphorylation were respectively estimated by western blot in response to $500 \mathrm{ng} \mathrm{PRL} / \mathrm{ml}$ after $10 \mathrm{~min}$ of treatment (A) and $500 \mathrm{ng}$ leptin/ml after $10 \mathrm{~min}$ of treatment following a pretreatment for $4 \mathrm{~h}$ in the presence of $500 \mathrm{ng}$ PRL/ml (B). Total STAT-5 and 3 were also determined. All experiments were performed in duplicate in two independent experiments. 
A

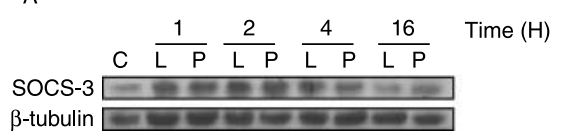

$\beta$-tubulin w

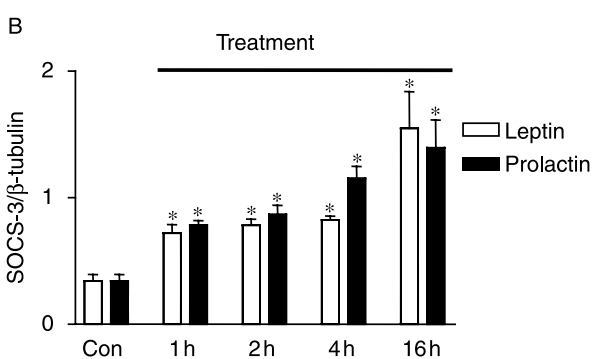

C

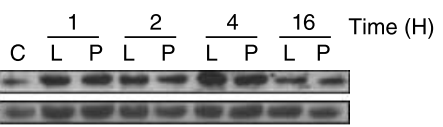

D

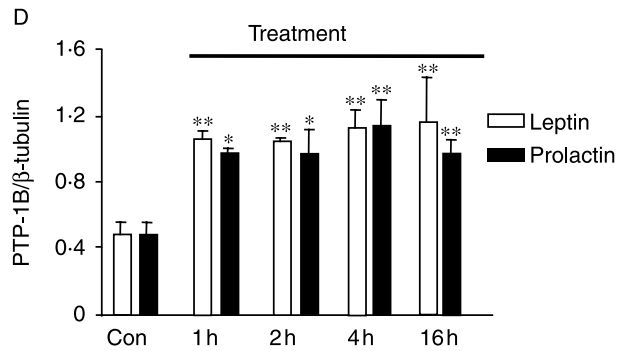

Figure 8 Leptin and PRL induce SOCS-3 and PTP-1B in CHO-ObRb/PRL-R cells. CHO cells stably co-expressing ObRb and PRL-R were starved and pre-treated with leptin or PRL $(500 \mathrm{ng} / \mathrm{ml})$ for various time periods. SOCS-3 (A and B) and PTP-1B (C) were estimated by western blot using specific antibodies, results are normalized to $\beta$-tubulin. SOCS- 3 and PTP-1B expression was measured in three independent experiments and the results expressed as mean \pm S.E.M.; ${ }^{*} P<0 \cdot 05$ and ${ }^{* *} P<0 \cdot 005$.

The possible cross-talk between leptin and PRL signaling pathways in the hypothalamus could have a physiological relevance, because we first showed that in the female rat hypothalamus both PRL-R and ObRb are expressed in the PVN. Interestingly, both the receptors co-localize in the same neurons. To our knowledge, this is the first evidence for such co-localization, which is a prerequisite for a possible cross-talk between both receptor signaling pathways. Moreover, by brain slice experiments, we showed that virgin females are sensitive to leptin, whereas lactating females are leptin resistant. To investigate this putative cross-regulation between $\mathrm{ObRb}$ and PRL-R, CHO cells stably co-transfected with PRL-R and ObRb were produced. In these cells, leptin and PRL clearly induced the phosphorylation of STAT-3 and 5 respectively. The phosphorylation of STAT-3 and 5 in response to leptin and PRL respectively increased over the first $30 \mathrm{~min}$ of incubation with a maximum being achieved after $10 \mathrm{~min}$ of treatment and then declined from $60 \mathrm{~min}$ of incubation. To mimic gestation or lactation hormonal environment, $\mathrm{CHO}-\mathrm{ObR}$ /PRL-R were subjected to PRL pre-treatment. In this condition, PRL-R signaling was completely abolished, as mirrored by a clear desensitization of PRL-R/STAT-5 signaling pathway in response to PRL stimulation. In contrast, the ObRb/STAT-3 signaling pathway was not affected by PRL pre-treatment and leptin still clearly increased STAT-3 phosphorylation. Similar results were obtained when cells were subjected to leptin overexposure. The ObRb/STAT-3 signaling pathway was completely inhibited and a clear leptin resistance is shown, whereas the PRL-R/STAT-5 signaling pathway was not affected and PRL sensitivity was not altered. Our results thus indicate the absence of cross-regulation between PRL-R and $\mathrm{ObRb}$. However, as this result could be attributed to an inappropriate ratio of PRL-R/ObRb expressed at the cell surface. $\mathrm{CHO}$ cells stably expressing ObRb were transfected with increased amount of PRL-R expressing vector. The $\mathrm{ObRb}$ /STAT-3 signaling pathway in those cells was also not affected by PRL overexposure, thus confirming our previous data and indicating the lack of a cross-desensitization between PRL-R and ObRb.

Interestingly, the common negative regulator SOCS-3 was induced by both leptin and PRL overexposure. Our results establish that the induction of SOCS-3 in response to long-term stimulation by leptin or PRL is only able to shut down the signaling of the inducer receptor. For instance, PRL treatment reduces PRL-R signaling but not leptin signaling and vice versa. These results contrast with previous studies that have described a negative cross-talk between interleukin-3 and interleukin-11 through the induction of SOCS-3 (Magrangeas et al. 2001). It is, however, shown that growth hormone $(\mathrm{GH})$ pre-treatment of adipocytes does not affect STAT-3 phosphorylation induced by cardiotropin-1a (CT1) or ciliary neutrophic factor (CNTF), which belong to interleukin-6 family but completely abolished GH-dependent STAT-5 phosphorylation (Zvonic et al. 2005). This is in good agreement with our findings, since GH induced SOCS-3 expression without affecting CT1 or CNTF signaling. Furthermore, we have also shown that both leptin and PRL long-term pre-treatment induced PTP-1B. To our knowledge, this is the first study to show such induction. Despite the increased PTP-1B following PRL exposure, ObRb signaling was not altered and this contrasts with previous studies (Lund et al. 2005) which have demonstrated that PTP-1B clearly reduced the phosphorylation of STAT- 3 and JAK- 2 in response to leptin. Taken together, our data suggest the absence of crosstalk between PRL-R and ObRb signaling pathways despite the significant induction of similar negative regulators such as SOCS-3 and PTP-1B. The hypothesis of intracellular compartmentalization could be a possible explanation suggesting that the induced negative regulator will only affect the inducer receptor. The compartmentalization has been 
suggested for several signaling pathways such as MAP kinases (Caunt et al. 2006), protein kinase A (Ghil et al. 2006), and tumor necrosis factor (TNF) receptor 1 signaling pathways (Hunter \& Nixon 2006).

If this concept is generalized in vivo, this should indicate that the hyperphagia observed during gestation and lactation can not be attributed to the inhibition of leptin signaling through the induction of SOCS-3, but is probably attributed to the direct effect of PRL on the expression of NPY at the hypothalamic level, as suggested previously (Freemark 2001). However, leptin resistance observed in these physiological states may have an additive effect by exacerbating food intake.

\section{Acknowledgements}

We thank Conseil Regional d'Ile de France for financial support (SESAME GRANT A01947), and we are grateful to Prof. C Bjorbaek for providing cDNA encoding for mouse ObRb. We also thank D Crépin for her technical assistance. The authors declare that there is no conflict of interest that would prejudice the impartiality of this scientific work.

\section{References}

Bjorbaek C, Votani S, da Silva B \& Flier JS 1997 Divergent signalling capacities of the long and short isoforms of the leptin receptor. Journal of Biological Chemistry 272 32686-32695.

Bole-Feysot C, Goffin V, Edery M, Binart N \& Kelly PA 1998 Prolactin (PRL) and its receptor: actions, signal transduction pathways and phenotypes observed in PRL receptor knockout mice. Endocrine Reviews 19 225-268.

Brogan RS, Mitchell SE, Trayhurn P \& Smith MS 1999 Suppression of leptin during lactation: contribution of the suckling stimulus versus milk production. Endocrinology 140 2621-2627.

Caunt CJ, Finch AR, Sedgley KR \& McArdle CA 2006 Seventransmembrane receptor signaling and ERK compartmentalization. Trends in Endocrinology and Metabolism 17 276-283.

Chehab FF, Lim ME \& Lu R 1996 Correction of the sterility defect in homozygous obese female mice by treatment with the human recombinant leptin. Nature Genetics 12 318-320.

Chen P \& Smith MS 2004 Regulation of hypothalamic neuropeptide Y messenger ribonucleic acid expression during lactation: role of prolactin. Endocrinology 145 823-829.

Chien EK, Hara M, Rovard M, Yano H, Phillipe M, Polonsky KS \& Bell GI 1997 Increase in serum leptin and uterine leptin receptor messenger RNA levels during pregnancy in rats. Biochemical and Biophysical Research Communications 237 476-480.

Denis RGP, Bing C, Naderali EK, Vernon RG \& Williams G 2003 Lactation modulates diurnal expression profiles of specific leptin receptor isoforms in the rat hypothalamus. Journal of Neuroendocrinology 178 225-232.

Douglas AJ, Johnstone LE \& Leng G 2007 Neuroendocrine mechanisms of change in food intake during pregnancy: a potential role for brain oxytocin. Physiology and Behavior 91 352-382.

Edery M, Jolicoeur C, Levi-Meyrueis C, Dusantier-Fourt I, Petridou B, Boutin JM, Lesueur L, Kelly PA \& Djiane J 1989 Identification and sequence analysis of a second form of prolactin receptor by molecular cloning of complementary DNA from rabbit mammary gland. PNAS $\mathbf{8 6}$ 2111-2116.

Freemark M 2001 Ontogenesis of prolactin receptors in the human foetus: role in foetal development. Biochemical Society Transactions 29 38-41.
Garcia MDC, Casanueva FF, Dieguez C \& Seranis RM 2000 Gestational profile of leptin messenger ribonucleic acid (mRNA) content in the placenta and adipose tissue in the rat, and regulation of the mRNA levels of the leptin receptor subtypes in the hypothalamus during pregnancy and lactation. Biology of Reproduction 62 698-703.

Gertler A, Simmons J \& Keisler DH 1998 Preparation and characterization of recombinant ovine obese protein (leptin). FEBS Letters 422 137-140.

Ghil S, Choi JM, Kim SS, Lee YD, Liao Y, Birnbaumer L \& Suh-Kim H 2006 Compartmentalization of protein kinase A signaling by the heterotrimeric G protein G0. PNAS 103 19158-19163.

Grattan DR 2002 Behavioural significance of prolactin signalling in the central nervous system during pregnancy and lactation. Reproduction $\mathbf{1 2 3}$ 497-506.

Grattan DR, Pi XJ, Andrews ZB, Augustine RA, Kokay IC, Summerfield MR, Todd B \& Bunn SJ 2001 Prolactin receptors in the brain during pregnancy and lactation: implications for behavior. Hormones and Behavior 40 115-124.

Grattan DR, Ladyman SR \& Augustine RA 2007 Hormonal induction of leptin resistance during pregnancy. Physiology and Behavior 91 366-374.

Grueso E, Rocha M \& Puerta M 2001 Plasma and cerebrospinal fluid leptin levels are maintained despite enhanced food intake in progesterone-treated rats. European Journal of Endocrinology 144 659-665.

Hoggard N, Mercer JG, Rayner DV, Moar K, Trayhurn P \& Williams LM 1997 Localization of leptin receptor mRNA splice variants in murine peripheral tissues by RT-PCR and in situ hybridization. Biochemical and Biophysical Research Communications 232 383-387.

Hunter I \& Nixon GF 2006 Spatial compartmentalization of tumor necrosis factor (TNF) receptor 1-dependent signaling pathways in human airway smooth muscle cell. Journal of Biological Chemistry 281 34705-34715.

Ihle JN 1995 Cytokine receptor signalling. Nature 377 591-594.

Kawai M, Yamaguchi M, Murakami T, Shima K, Murata Y \& Kishi K 1997 The placenta is not the main source of leptin production in pregnant rat: gestational profile of leptin in plasma and adipose tissues. Biochemical and Biophysical Research Communications 240 798-802.

Kitawaki J, Koshiba H, Ishihara H, Kusuki I, Tsukamoto K \& Honjo H 2000 Expression of leptin receptor in human endometrium and fluctuation during the menstrual cycle. Journal of Clinical Endocrinology and Metabolism 85 1946-1950.

Lage M, Garcia-Mayor R, Tomé MA, Cordido F, Valle-Inclan F, Considine RV, Caro JF, Dieguez C \& Casanueva FF 1999 Serum leptin levels in women throughout pregnancy and postpartum period and in women suffering spontaneous abortion. Clinical Endocrinology 50 211-216.

Lewandowski K, Horn R, O'Callaghan CJ, Dunlop D, Medley GF, O'Hare P \& Brabant G 1999 Free leptin, bound leptin, and soluble leptin receptor in normal and diabetic pregnancies. Journal of Clinical Endocrinology and Metabolism 84 300-306.

Lund IK, Hansen JA, Andersen HS, Moller NPH \& Billestrup N 2005 Mechanism of protein tyrosine phosphatase $1 \mathrm{~B}$-mediated inhibition of leptin signaling. Journal of Molecular Endocrinology 34 339-351.

Magrangeas F, Boisteau O, Denis S, Jacques Y \& Minvielle S 2001 Negative cross-talk between interleukin-3 and interleukin-11 is mediated by suppressor of cytokine signalling-3 (SOCS-3). Biochemical Journal 353 223-230.

Masuzaki H, Ogawa Y, Sagwa N, Hosoda K, Matsumoto T, Mise H, Nishimura H, Yoshimasa Y, Tanaka I, Mori Tet al. 1997 Nonadipose tissue production of leptin: leptin as a novel placenta-derived hormone in humans. Nature Medicine 3 1029-1033.

Mukherjea R, Castonguay TW, Douglas LW \& Moser-Veillon P 1999 Elevated leptin concentrations in pregnancy and lactation: possible role as modulator of substrate utilization. Life Sciences 65 1183-1193.

Rosso P 1987 Regulation of food intake during pregnancy and lactation. Annals of the New York Academy of Sciences 25 683-691.

Sauvé D \& Woodside B 2000 Neuroanatomical specificity of prolactininduced hyperphagia in virgin female rats. Brain Research 868 306-314.

Seeber RM, Smith JT \& Waddell BJ 2002 Plasma leptin-binding activity and hypothalamic leptin receptor expression during pregnancy and lactation in rat. Biology of Reproduction 66 1762-1767. 
Shirley B 1984 The food intake of rats during pregnancy and lactation. Laboratory Animal Science 34 169-172.

Tomimatsu T, Yamaguchi M, Murakami T, Ogura K, Sakata M, Mitsuda N, Kanzaki T, Kurachi H, Irahara M, Miyake A et al. 1997 Increase of mouse leptin production by adipose tissue after midpregnancy: gestational profile of serum leptin concentration. Biochemical and Biophysical Research Communications 240 213-215.

Vacher CM, Frétier P, Créminon C, Calas A \& Hardin-Pouzet H 2002 Activation by serotonin and noradrenaline of vasopressin and oxytocin expression in the mouse paraventricular and supraoptic nuclei. Journal of Neuroscience 22 1513-1522.

Woodside B 2007 Prolactin and the hyperphagia of lactation. Physiology and Behavior 91 375-382.
Zamorano PL, Mahesh VB, De Sevilla LM, Chorich LP, Bhat GK \& Brann DW 1997 Expression and localization of the leptin receptor in endocrine and neuroendocrine tissues of the rat. Neuroendocrinology 65 223-228.

Zvonic S, Baugh JE, Arbour-Reily P, Mynatt RL \& Stephens JM 2005 Cross-talk among gp130 cytokines in adipocytes. Journal of Biological Chemistry 280 33856-33863.

Received in final form 17 August 2007

Accepted 5 September 2007

Made available online as an Accepted Preprint 5 September 2007 